\title{
THE EFFICACY OF GLUCOSAMINE AND CHONDROITIN SULFATE IN THE TREATMENT OF OSTEOARTHRITIS: ARE THESE SACCHARIDES DRUGS OR NUTRACEUTICALS?
}

\author{
Vilím Šimánek ${ }^{\mathrm{a}}$, Vladimír Křen ${ }^{\mathrm{b}}$, Jitka Ulrichováa, Jiří Gallo ${ }^{c *}$ \\ ${ }^{a}$ Institute of Medical Chemistry and Biochemistry, Faculty of Medicine, Palacký University, Hněvotínská 3, 77515 \\ Olomouc \\ ${ }^{b}$ Institute of Microbiology, Academy of Sciences of the Czech Republic, Videñská 1083, 142 20, Prague \\ c Clinic of Orthopaedics, Teaching Hospital, Palacký University, I. P. Pavlova 6, 77520 Olomouc, Czech Republic \\ e-mail: jiri.gallo@volny.cz
}

Received: June 2, 2005; Accepted: June 30, 2005

Key words: Osteoarthritis/Mediators/Glycosaminoglycan metabolism/Intestinal absorption/Intestinal degradation/Chondrocytes/Drug/Food Supplement/Safety/SYSADOA

This review summarizes recent knowledge on the efficacy of glucosamine (GS) and/or chondroitin sulfate (CS) in the therapy of mild to moderate osteoarthritis (OA). OA, the most common joint disease is a significant source of disability, quality of life impairment and a considerable burden to any health care system. In the Czech Republic, glucosamine sulfate (GS) and chondroitin sulfate (CS) are available both as prescription drugs and as foof supplements. Based on available data both are useful in the earlier stages of OA when combined with other modalities such as weight loss and exercises. They appear to relieve pain and improve range of the joint motion. In addition, they also display mild anti-inflammatory effects. However, controversy still exists over their ability to change significantly the natural history of the osteoarthritic joint. This effect is not easy to demonstrate for any other treatment modalities apart from joint replacement. Monitoring the cure efficacy by X-ray has been recently criticised and hence future techniques are anticipated for this reason. Further, long-term oral administration is required to obtain slightly increased levels of GS and/or CS in human blood. Both reviewed saccharides are well tolerated with negligible adverse reactions. In conclusion, the authors suggest that GS and CS should be classified as food supplements only.

\section{INTRODUCTION}

Osteoarthritis (OA) is the most common disorder of the synovial joints in middle aged and older people. ${ }^{1}$ It is characterised predominantly by a focal or global loss of articular cartilage, bone changes and an imbalance in inflammatory and non-inflammatory pathways including proteolysis of aggrecans and collagens combined with distortion of their synthesis by chondrocytes. ${ }^{2}$ It is a source of great morbidity, impaired quality of life in affected individuals as well as a significant burden to any health care system. ${ }^{1}$ It is estimated that more than one third of people over 45 years complain of OA-related symptoms.

The leading symptoms of the OA are pain, stiffness and decreasing functional capacity of the affected joint. ${ }^{3}$ The natural history of the disease may vary from a very slow process to a progressive one, where the joint is severely eroded over several months. Fortunately the former is more frequent. On the other hand, spontaneous resolution of a previously osteoarthritic joint has only been mentioned anecdotally. ${ }^{4}$ Recently, OA has been interpreted as manifestation of a complex disease with a complicated structure of gene dispositions. ${ }^{5}$
In the past decades, surgical and conservative protocols have been developed to treat osteoarthritis. ${ }^{6}$ These are targeted at pain control, inhibition of inflammatory cytokines and proteolytic enzyme activity, free radical release damping, increasing chondrocyte number and function, modification of mechanical conditions in favour of the affected cartilages, etc. However, if the final goal is to fully restore the structural and functional properties of the original tissues, none of the above is adequately reliable. ${ }^{7}$ Glucosamine (GS) and chondroitin sulfate (CS) are routinely used in practice. The aim of this paper is to review their efficacy and safety in the treatment of OA.

\section{The natural role of GS and CS in cartilage structure}

Articular cartilages represent a highly organized mixture of chondrocytes, Type II collagen, proteoglycans and water that develop as part of endoskeleton growth and mature under the influence of functional loading. ${ }^{8}$ Chondrocytes produce components of the extracellular matrix and regulate the cartilage metabolism similarly to cells in other connective tissues. ${ }^{9}$ In this context, the number and functionality of chondrocytes guarantee the anatomical and tribological features of the cartilage.${ }^{10}$ However, 
the mitotic and synthetic activities of these cells decline with age. ${ }^{11}$

The macromolecular composition of extracellular matrix, where Type II collagens and aggrecans are the most important and abundant, is a key factor defining the physicochemical properties of the cartilage. ${ }^{12}$ Aggrecans are large molecules consisting of a central protein core with several distinct domains and with their different functions..$^{13}$ Chondroitin sulfate and the N-terminal hyaluronan-binding (G1) part belong to the most important domains. Glycosaminoglycans are linear acidic polysaccharides containing disaccharide repeat units of D-glucuronate/L-iduronate/D-galactose linked to sulphonated $N$-acetylglucosamine/ $N$-acetylgalactosamine. Such complexes are naturally synthesized in each joint resulting in high local concentrations.

\section{Chondroitin sulfate}

Chondroitin sulfate consists of an alternating sequence of D-glucuronate and $N$-acetyl-D-galactosamine-4/6-sulfate residues linked through alternating bonds (Scheme 1). It is an essential component of the connective tissue extracellular matrix including the hyaline cartilage, providing its elasticity and other functions. CS belongs to a heteroge- neous family of glycosaminoglycans with a relatively high molecular weight and charge density. ${ }^{9}$ The CS is absorbed in the small intestine in low amounts $(<10 \%)$ in the intact form, probably by the mechanism of endocytosis. In the distal gastrointestinal tract, the $\mathrm{CS}$ acts as a prebiotic and is degraded by the enzymes in the intestinal flora to di- and monosaccharides. ${ }^{14}$ These substances help to maintain constant levels of proteoglycan precursors and also supplement the $N$-acetylgalactosamine. Numerous studies have demonstrated the efficacy and availability of CS in experimental and human tissues. ${ }^{14-16} \mathrm{CS}$ is usually manufactured from bovine or porcine cartilaginous material and also from shark cartilage. Various CS formulations are strongly influenced by the structure and characteristics of raw material origin. ${ }^{17}$ The recommended dose for long term usage is $800 \mathrm{mg}$ per day.

\section{Glucosamine}

GS is an amino monosaccharide, which participates in the constitution of glycosaminoglycans, a major class of extracellular complex polysaccharides. The raw material is derived from chitin, a biopolymer present in the exoskeleton of marine intervertebrate animals. ${ }^{18}$ There may be significant differences in purity and other phar-

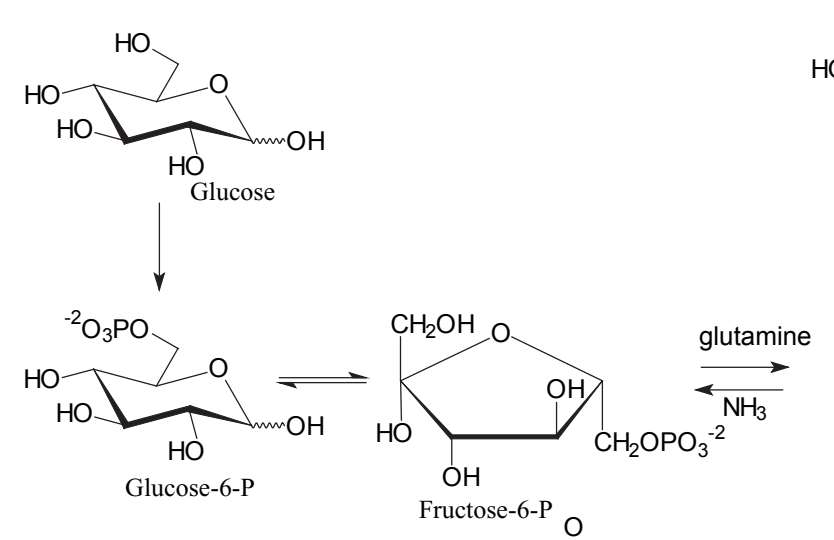

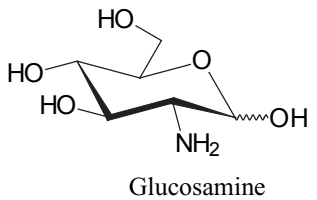

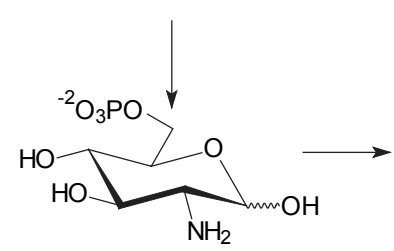

Glucosamine-6-P

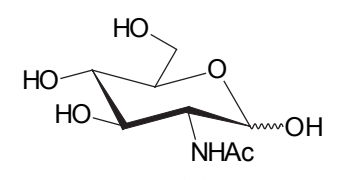

$N$-Acetylglucosamine

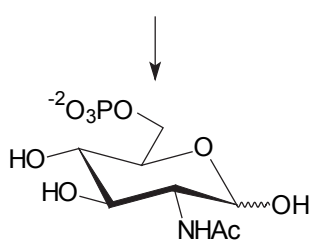

$N$-Acetylglucosamine-6-P

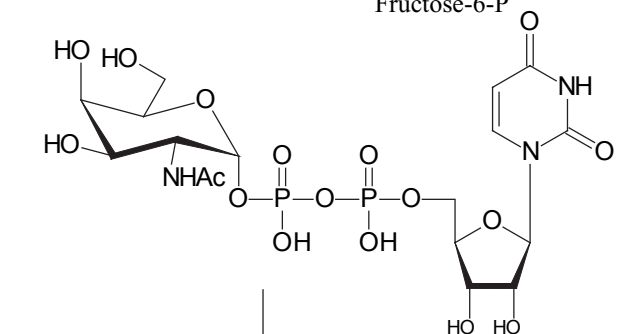

UDP- $N$-Acetylgalactosamine

$\downarrow$

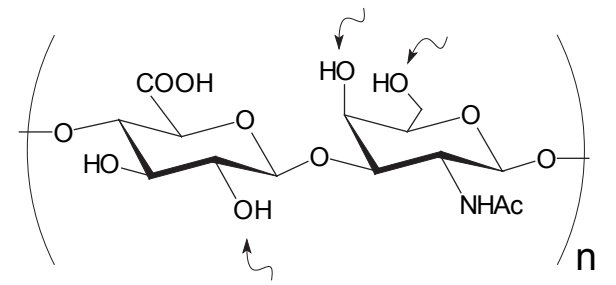

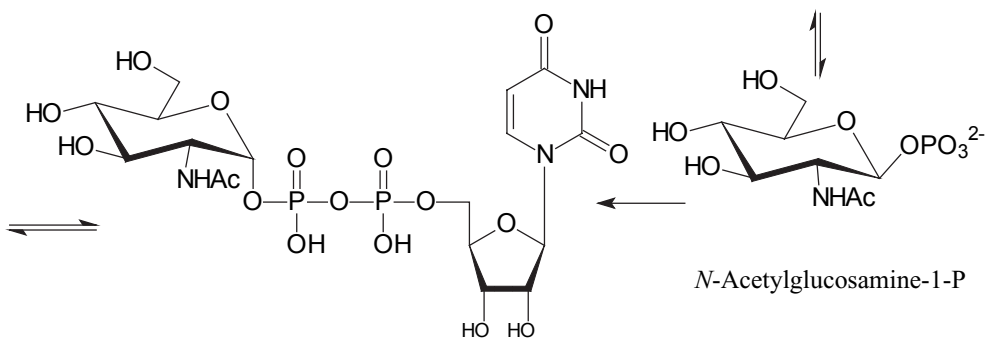

UDP- $N$-Acetylglucosamine

CHONDROITIN - arrows - potential sites of sulfatation

Scheme 1. Biosynthesis of chondroitin. 

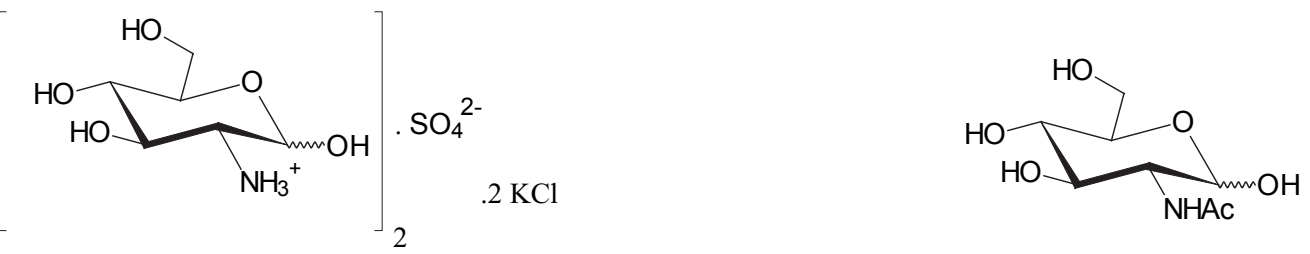

Scheme 2. Glucosamine sulfate (co-crystallized with $2 \mathrm{KCl}$ ) and 2-Acetamido-2-deoxy-D-glucopyranoside ( $N$-Acetylglucosamine, GlcNAc).

maceutical features among products even though the GS is processed only by a few companies..$^{19}$ Glucosamine sulfate, glucosamine hydrochloride and $N$-acetyl-glucosamine (Scheme 2) are commonly used alone or as part of the mixtures produced by the pharmaceutical industry. GS is usually taken orally despite having no active intestinal transport. ${ }^{20,21}$ The recommended dose for long term usage is $1500 \mathrm{mg}$ per day. According to Anderson et al. ${ }^{20}$ daily concentrations of GS in the serum can reach $0.06 \mathrm{mM}$ when a routine dosage of $23.1 \mathrm{mg} / \mathrm{kg}$ body weight is administered. It seems unlikely that such low levels would interfere with sugar metabolism. In contrast to Anderson, other authors recommend special care when GS is administrated to patients with diabetes Type II (ref. ${ }^{22}$ ). In addition, $N$-acetylglucosamine, a metabolic product of GS, enhances basal and fMLP-induced ( $N$-formyl-methionylleucyl-fenylalanine) motility in neutrophils by modification of serine/threonine residues on cytoplasmatic and nuclear proteins. ${ }^{23}$ This O-GSAc modification is thought to play a role in the regulation of different signal transductions.

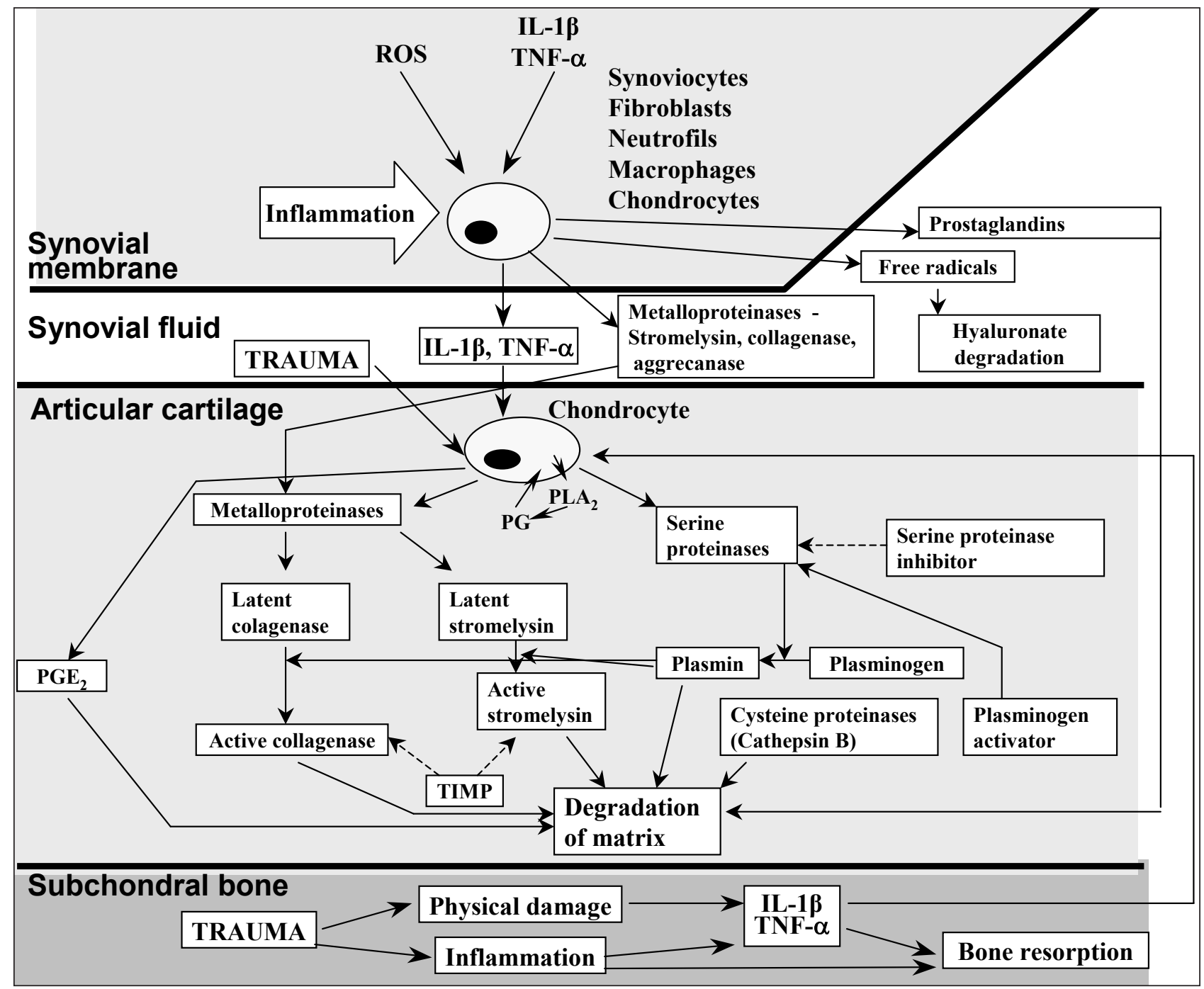

Fig. 1. The current concept of osteoarthritic cascade of events (ROS - reactive oxygen species, TIMP - tissue inhibitors of metalloproteinases). 
Suggested therapeutic action for GS and CS

There is suspected chondrocyte insufficiency and/or apoptosis in OA resulting in the inability to remodel effectively the extracellular matrix and repair local cartilage defects. ${ }^{3} \mathrm{OA}$ is associated with increasing levels of matrix metalloproteinases - collagenases, aggrecanases, stromelysin etc. ${ }^{24}$ Simultaneously aseptic inflammation of synovial tissues is present with changes in joint fluid composition that may further impair cartilage metabolism and its wear. ${ }^{25}$ Interleukin $1 \beta$ and TNF- $\alpha$ are considered to be the most prominent inflammatory cytokines to participate in osteoarthritic progression. ${ }^{2,26}$ Both induce NO release through mesenchymal cells. NO as well as other free radicals may increase chondrocyte vulnerability and apophosis. In concert, all these factors strongly distort joint homeostasis in terms of cartilage loss, capsule thickening and a series of ligament, muscle and bone changes (Fig. 1).

Glucosamine and chondroitin sulfate are symptomatic slow-acting drugs for osteoarthritis (SYSADOA). These substances are characterised by both a several week delay in improvement of OA symptoms and by a carryover effect of that improvement. ${ }^{6}$ The rationale for their usage is based on a general belief that osteoarthritis is associated with a local deficiency in some key natural substances. Therefore, it is assumed that they work as a "building box" for cartilage extracellular matrix repair. ${ }^{21,27}$ Increasing proteoglycan synthesis by chondrocytes has been suggested as another mechanism of CS action. ${ }^{28}$ In addition, CS may inhibit the activity of degradative enzymes. ${ }^{28,29}$ Implied "anti-osteoarthritic" activities of GS and CS are summarized in the table 1.

\section{Evidence for GS and CS usage}

In fact, a supplementation approach looks rather simplistic against the background of a not as yet fully under- stood disease pathophysiology. Despite this, many studies have been conducted to demonstrate significant pain relief and functional improvement after GS or CS therapy in OA when compared to either placebo or NSAIDs, using randomized controlled trials (RCTs) and metaanalyses. ${ }^{19,28,30,31}$ Unfortunately, the majority of studies were sponsored by the manufacturers and/or have methodological weaknesses. ${ }^{19}$ As a result, one may conclude that there is evidence for mild to moderate improvement of symptomes due to GS and CS. On the other hand, this does not exclude the possibility that they bring positive effects to many individuals, and, compared to NSAIDs they have a very low risk of adverse reactions. ${ }^{32}$ Nevertheless, many questions remain to be answered.

One of the most representative studies on the influence of CS/GS on knee osteoarthritis was begun in February 2000 at the National Institutes of Health (NIH) in the USA. The patients were randomly assigned to receive either (1) GS alone, (2) CS alone, (3) GS and CS in combination, (4) celecoxib (Celebrex), or (5) a placebo. Overall 13 research centres across the USA participated in this study under the coordination of the University Utah School of Medicine. However, the results of this multicenter and well-designed clinical trial are not available up to the present time. ${ }^{33}$

Controversy still exists over the structure-modifying effect of a GS or CS medication on the cartilage matrix. ${ }^{34}$ Long-term use of GS and/or CS has been suggested on the basis of systematic reviews of RCTs or meta-analysis. ${ }^{35,36}$ However, the main weaknesses of the analyzed RCTs are the lack of standardization of the OA diagnosis and a questionable outcome assessment. Unsuitability of joint space width measurement has been criticised repeatedly, and future techniques are expected for measurement of the true osteoarthritic regression. ${ }^{37-39}$ As a result, the structure-modifying effect still remains unproved for both

Table 1. Parameters used for assessment of glucosamine, $N$-acetylglucosamine and chondroitin sulfate "anti-osteoarthritic" activities in vitro/in vivo.

\begin{tabular}{|l|c|c|c|c|}
\hline Parameter & GS & N-AcetylGS & CS & Experiment \\
\hline Blood level of GS & $\uparrow$ & & & in vivo \\
\hline Blood level of CS & & & $\uparrow$ & in vivo \\
\hline UDP- $N$-acetylGS & $\uparrow$ & & & in vitro \\
\hline NO production & $\downarrow$ & $\downarrow$ & & in vitro \\
\hline PLA & $\downarrow$ & & $\downarrow$ & in vitro/in vivo \\
\hline mRNA of aggrecan and perlecan core proteins $^{2}$ & $\uparrow$ & & & in vitro \\
\hline Collagenase activity & $\downarrow$ & & $\downarrow$ & in vitro/in vivo \\
\hline IL-1 $\beta$ & $\downarrow$ & $\downarrow$ & & in vitro \\
\hline TNF- $\alpha$ & & $\downarrow$ & & in vitro \\
\hline MMP activity & $\downarrow$ & & $\downarrow$ & in vitro \\
\hline Proteoglycan synthesis & & & $\uparrow$ & in vitro \\
\hline COX-2 activity & & $\downarrow$ & & in vitro \\
\hline
\end{tabular}

Table excludes studies with limited statistical significance. $\downarrow \uparrow$ - Decreased/increased, COX-2 - Cyclooxygenase-2, MMP - Matrix metalloproteinase, IL-1 $\beta$ - Interleukin-1 $\beta$, PLA $_{2}$ - Phospholipase $\mathrm{A}_{2}$, TNF- $\alpha$ - Tumor necrosis factor $\alpha$, 
Table 2. Drugs and selected dietary supplements for osteoarthritis on the Czech market.

\begin{tabular}{|c|c|}
\hline Preparation & Active components (mg) in pill/sac \\
\hline CONDROSULF $400^{\mathrm{a}}$ & CS (400) \\
\hline CONDROSULF $800^{\mathrm{a}}$ & CS (800) \\
\hline DONA $^{\mathrm{a}}$ & GS (1500) \\
\hline HYALGAN syringe $^{\mathrm{a}}$ & $\mathrm{HUNa}^{\mathrm{b}}$ \\
\hline SYNVISC syringe $^{\mathrm{a}}$ & $\mathrm{HUNa}^{\mathrm{b}}(10 / 20)$ \\
\hline MOBILIN $^{c}$ & GS (1500), CS (50), CH 50) \\
\hline ARTRYN $^{c}$ & GS (800), CS (25), CH (175) \\
\hline ARTHROSTOP RAPID ${ }^{\mathrm{c}}$ & GS $(533,3)$, CS $(200)$ \\
\hline GELACTIV $^{\mathrm{c}}$ & GS (500), CS (400), CH, MSM (200) \\
\hline GS-CS-MSM $3000^{\circ}$ & GS (500), CS (200), MSM (300) \\
\hline GS-CONDRO $^{c}$ & GS (400), CH (200) \\
\hline GS-CONDROFORTE $^{c}$ & GS (800), CH (200) \\
\hline PROENZI 3c & GS (500), CS (200), MSM (300) \\
\hline PROENZI PREMIUM FORTE $2700^{\circ}$ & GS (500), CS (400) \\
\hline
\end{tabular}

${ }^{\mathrm{a}}$ Drug on prescription, ${ }^{\mathrm{b}} \mathrm{In} 1 \mathrm{ml}$, ${ }^{\mathrm{c} F o o d}$ supplement, CS - Chondroitin sulfate, GS - Glucosamine sulfate, HUNa - Sodium hyaluronate, $\mathrm{CH}$ - Collagene hydrolysate MSM - Methyl sulfonylmethane

reviewed substances. In addition, long-term monitoring of all patients involved in clinical studies and their appropriate medication may be also difficult. ${ }^{40}$ Moreover, different natural histories of the disease and the debatable mechanism of the CS and GS actions have to be considered.

\section{Drugs or nutraceuticals?}

A plethora of drugs and food supplements consisting of glucosamine and chondroitin sulfate are on the market in the Czech Republic (Table 2). Both GS and CS drugs and supplements are produced under similar if not the same conditions given to GMP, ISO standards, and internal audits. However, food supplements do not need approval from the State Institute for Drug Control. We believe that this mini-review shows that the potential difference between the above products is not significant enough to justify the existence of two controversial terms for one single substance (food supplements vs drugs). Moreover, it may be alleged that extensive prescription of the reviewed substances will not relieve the economic burden of the current treatment strategies for osteoarthritic joints.

\section{CONCLUSION}

Both CS and GS are clearly defined by their origin, chemical structure and expected efficacy. They exhibit a low risk of adverse effects. Based on the available data there is no rationale to distinguish between drugs and nutraceuticals with regard to the reviewed compounds (providing that the recommended dose and adequate purity are respected). As a result, we prefer the term food supplements to drug. In addition, this paper supports wider usage of the reviewed substances in earlier and moderate stages of osteoarthritis.

\section{ACKNOWLEDGEMENTS}

The authors have no conflict of interest that is directly relevant to the content of this review.

This work was supported by Ministry of Education grant MSM 6198959216.

\section{REFERENCES}

1. Buckwalter JA, Saltzman C, Brown T. (2004) The impact of osteoarthritis: implications for research. Clin Orthop Relat Res 427 Suppl, S6-15.

2. Goldring MB, Berenbaum F. (2004) The regulation of chondrocyte function by proinflammatory mediators: prostaglandins and nitric oxide. Clin Orthop Relat Res 427 Suppl, S37-46.

3. Dieppe PA, Lohmander LS. (2005) Pathogenesis and management of pain in osteoarthritis. Lancet 365(9463), 965-73.

4. Guyton GP, Brand RA. (2002) Apparent spontaneous joint restoration in hip osteoarthritis. Clin Orthop Relat Res 404, 302-7.

5. Loughlin J. (2005) The genetic epidemiology of human primary osteoarthritis: current status. Expert Rev Mol Med 7, 1-12.

6. Fajardo M, Di Cesare PE. (2005) Disease-modifying therapies for osteoarthritis: current status. Drugs Aging 22, 141-61.

7. Buckwalter JA, Martin JA. (2004) Sports and osteoarthritis. Curr Opin Rheumatol 16, 634-9.

8. Buckwalter JA, Martin J, Mankin HJ. (2000) Synovial joint degeneration and the syndrome of osteoarthritis. Instr Course Lect 49, 481-9.

9. Tuan RS. (2004) Biology of developmental and regenerative skeletogenesis. Clin Orthop Relat Res 427 Suppl, S105-17.

10. Baker CL, Ferguson CM. (2005) Future treatment of osteoarthritis. Orthopedics 28 (2 Suppl), S227-34.

11. Aigner T, Rose J, Martin J, Buckwalter J. (2004) Aging theories of primary osteoarthritis: from epidemiology to molecular biology. Rejuvenation Res 7, 134-45.

12. Eyre DR. (2004) Collagens and cartilage matrix homeostasis. Clin Orthop Relat Res 427 Suppl, S118-22.

13. Nagase H, Kashiwagi M. (2003) Aggrecanases and cartilage matrix degradation. Arthritis Res Ther 5, 94-103. 
14. Barthe L, Woodley J, Lavit M, Przybylski C, Philibert C, Houin G. (2004) In vitro intestinal degradation and absorption of chondroitin sulfate, a glycosaminoglycan drug. Arzneimittelforschung 54, 286-92.

15. Adebowale A, Du J, Liang Z, Leslie JL, Eddington ND. (2002) The bioavailability and pharmacokinetics of glucosamine hydrochloride and low molecular weight chondroitin sulfate after single and multiple doses to beagle dogs. Biopharm Drug Dispos 23, 217-25.

16. Volpi N, Maccari F. (2005) Microdetermination of chondroitin sulfate in normal human plasma by fluorophore-assisted carbohydrate electrophoresis (FACE). Clin Chim Acta 356, 125-33.

17. Volpi N. (2004) The pathobiology of osteoarthritis and the rationale for using the chondroitin sulfate for its treatment. Curr Drug Targets Immune Endocr Metabol Disord 4, 119-27.

18. Zhu X, Cai J, Yang J, Su Q. (2005) Determination of glucosamine in impure chitin samples by high-performance liquid chromatography. Carbohydr Res, 340, 1732-1738.

19. Towheed T, Maxwell L, Anastassiades T, Shea B, Houpt J, Robinson V, Hochberg M, Wells G. (2005) Glucosamine therapy for treating osteoarthritis. Cochrane Database Syst Rev 2, CD002946.

20. Anderson JW, Nicolosi RJ, Borzelleca JF. (2005) Glucosamine effects in humans: a review of effects on glucose metabolism, side effects, safety considerations and efficacy. Food Chem Toxicol 43 , 187-201.

21. Tiraloche G, Girard C, Chouinard L, Sampalis J, Moquin L, Ionescu M, Reiner A, Poole AR, Laverty S. (2005) Effect of ora glucosamine on cartilage degradation in a rabbit model of osteoarthritis. Arthritis Rheum 52, 1118-28.

22. Adams ME. (1999) Hype about glucosamine. Lancet 354(9176), 353-4.

23. Kneass ZT, Marchase RB. (2005) Protein O-GlcNAc modulates motility-associated signaling intermediates in neutrophils. J Biol Chem 280, 14579-85.

24. Mix KS, Sporn MB, Brinckerhoff CE, Eyre D, Schurman DJ. (2004) Novel inhibitors of matrix metalloproteinase gene expression as potential therapies for arthritis. Clin Orthop Relat Res 427 Suppl, S129-37.

25. Carter DR, Beaupre GS, Wong M, Smith RL, Andriacchi TP, Schurman DJ. (2004) The mechanobiology of articular cartilage development and degeneration. Clin Orthop Relat Res 427 Suppl, S69-77.

26. Goldring SR, Goldring MB. (2004) The role of cytokines in cartilage matrix degeneration in osteoarthritis. Clin Orthop Relat Res 427 Suppl, S27-36.
27. Curtis CL, Harwood JL, Dent CM, Caterson B. (2004) Biological basis for the benefit of nutraceutical supplementation in arthritis. Drug Discov Today 9, 165-72.

28. Michel BA, Stucki G, Frey D, De Vathaire F, Vignon E, Bruehlmann P, Uebelhart D. (2005) Chondroitins 4 and 6 sulfate in osteoarthritis of the knee: a randomized, controlled trial. Arthritis Rheum 52, 779-86.

29. Cibere J, Thorne A, Kopec JA, Singer J, Canvin J, Robinson DB, et al. (2005) Glucosamine sulfate and cartilage Type II collagen degradation in patients with knee osteoarthritis: Randomized discontinuation trial results employing biomarkers. J Rheumatol 32, 896-902.

30. Cibere J, Kopec JA, Thorne A, Singer J, Canvin J, Robinson DB, et al. (2004) Randomized, double-blind, placebo-controlled glucosamine discontinuation trial in knee osteoarthritis. Arthritis Rheum 51, 738-45.

31. Leeb BF, Schweitzer H, Montag K, Smolen JS. (2000) A metaanalysis of chondroitin sulfate in the treatment of osteoarthritis. J Rheumatol 27, 205-11.

32. Danao-Camara T. (2000) Potential side effects of treatment with glucosamine and chondroitin. Arthritis Rheum 43: 2853.

33. Glucosamine/Chondroitin Arthritis Intervention Trial. National Institutes of Health. http://nccam.nih.gov/news (June 15, 2005).

34. Moskowitz RW, Hooper M. (2005) State-of-the-art. Disease-modifying Osteoarthritis Drugs. Curr Rheumatol Rep 7, 15-21.

35. Poolsup N, Suthisisang C, Channark P, Kittikulsuth W. (2005) Glucosamine long-term treatment and the progression of knee osteoarthritis: systematic review of randomized controlled trials. Ann Pharmacother, in press.

36. Richy F, Bruyere O, Ethgen O, Cucherat M, Henrotin Y, Reginster JY. (2003) Structural and symptomatic efficacy of glucosamine and chondroitin in knee osteoarthritis: a comprehensive meta-analysis. Arch Intern Med 163, 1514-22.

37. Abadie E, Ethgen D, Avouac B, Bouvenot G, Branco J, Bruyere O, et al. (2004) Recommendations for the use of new methods to assess the efficacy of disease-modifying drugs in the treatment of osteoarthritis. Osteoarthritis Cartilage 12, 263-8.

38. Altman RD. (2004) Measurement of structure (disease) modification in osteoarthritis. Osteoarthritis Cartilage 12 Suppl A, S6976.

39. Dougados M. (2005) How can one develop disease-modifying drugs in osteoarthritis? Curr Rheumatol Rep 7, 22-8.

40. Mazzuca SA, Brandt KD, Katz BP, Lane KA, Bradley JD, Heck LW, et al. (2004) Subject retention and adherence in a randomized placebo-controlled trial of a disease-modifying osteoarthritis drug. Arthritis Rheum 51, 933-40. 